\section{New and Emerging Medications}

When I think back to the drugs I used when I started anesthesia practice, most are totally different from what I use on a daily basis currently. In many ways, these newer drugs were the major factor in the tremendous growth of office-based surgery and anesthesia. By allowing clear and rapid recovery from even deeper levels of anesthesia, patients could be safely discharged in a relatively short time period.

There was a time, before the early 1970s, when general anesthesia for medical procedures outside the hospital was almost unheard of. Nearly all hospital patients were admitted the day before surgery with general anesthesia and were discharged the following day. Essentially, the only anesthesia providers in the office setting at that time were oral surgeons! Early on, this consisted of inhalation anesthesia, primarily with nitrous oxide, trichloroethylene, in the late 1950s halothane, and later still enflurane and isoflurane. Intubated and nasopharyngeal administration of inhalation agents were commonly used by oral surgeons early in the 20th century. Inhalation agents have since advanced significantly with the introduction of desflurane, still the most rapid onset/offset potent inhalation agent with a very low fat solubility, which was introduced in 1992. In controlled studies in which anesthesia drugs are turned off at the end of surgery, time to eye opening was faster with desflurane than even propofol, although total recovery times with hospital protocols did not vary as much. Of course, sevoflurane supplanted halothane for inhalation induction.

By the 1940s, however, many oral surgeons and other dentists had begun to switch from inhalation to primarily intravenous agents. With the introduction of more intravenous agents, oral surgeons then became the pioneers of intravenous office-based anesthesia and surgery starting with the introduction of intravenous thiopental by John Lundy, MD, at the Mayo Clinic in 1934. Dr Adrian Hubbell, an oral surgery graduate of Mayo Clinic, popularized intravenous thiopental anesthesia in office oral surgery, including his development of a foot-operated pump nicknamed the "Hubbell Bubble" to inject more thiopental when needed while simultaneously operating. The Jorgensen moderate sedation technique using pentobarbital, scopolamine, and meperidine was introduced in 1945 as an alternative to general anesthesia. Methohexital (Brevital) eventually supplanted thiopental in oral surgery practice because of the faster recovery profile. For those of us who have been

(C) 2016 by the American Dental Society of Anesthesiology practicing for more than 15 years, we remember the days of methohexital with the hiccups, shaking, laryngospasms, and prolonged recovery periods. Of course, when propofol was introduced in 2001, the era of clear and rapid awakening from intravenous general anesthesia began. It is hard to remember the prepropofol days now!

The advent of esterase metabolized drugs further improved recovery profiles for intravenous drugs. Remifentanil allows for continuous infusions of even high doses of opioid with predictable recovery of breathing in less than 15 minutes. Lower infusion doses allow for spontaneous respiration intraoperatively while intubated or with a natural airway. When combined with propofol, a decreased dose of that drug further improves recovery characteristics. Of course, there were other short-acting esterase metabolized drugs commonly used in anesthetic practice before remifentanil such as esmolol, succinylcholine, and mivacurium (since discontinued).

Now, there is current research into a new esterase metabolized benzodiazepine: remimazolam. This drug combines the pharmacodynamics of midazolam with the esterase metabolism pharmacokinetics of remifentanil. Phase 3 Food and Drug Administration trials are soon to be completed, so approval may be a short number of years away. The medication will need to be administered by continuous intravenous infusion. One of the benefits of this drug is that, at the proper dose, it is more likely that there will be very reliable amnesia, even for long procedures, while still maintaining the patient at the moderate sedation level. If oversedation occurs, in the elderly, for instance, it may be possible to terminate the infusion or decrease the dose, with rapid return to the appropriate, and safe, sedative level. This may be a game changer for moderate-sedation dentists as well as dentists providing deep sedation and general anesthesia. Many more patients could tolerate longer dental procedures if amnesia were more reliable with moderate sedation techniques and the recovery profile were improved. Safety of intravenous dental sedation should improve, and patient satisfaction should increase.

Another recently introduced drug in the United States that may have implications in office-based anesthesia is sugammadex (Bridion). Sugammadex reliably reverses neuromuscular blockade induced by rocuronium or vecuronium. It is a modified cyclodextrin with a lipophilic interior and hydrophilic exterior that encapsulates these steroidal neuromuscular blocking agents, rendering them unavailable to interact at the neuromuscular junction. In SSDI 0003-3006(16) 
dental office-based general anesthesia, paralysis is frequently not used for intubation, particularly when propofol-remifentanil mixtures and/or deep sevoflurane anesthesia are generally very reliable in obtaining good intubating conditions. However, for deep sedation and general anesthesia providers, succinylcholine has been a required neuromuscular blocking agent for the treatment of laryngospasm and for emergency intubation. Onset of paralysis with a full $1-1.5 \mathrm{mg} / \mathrm{kg}$ intubating dose, as might be used for emergency intubation or treatment of laryngospasm with low oxygen saturation, is rapid (within 30-60 seconds), with recovery starting at 3 minutes and complete in 8-15 minutes. For those with current intubation experience who can rapidly secure the airway, a dose of $0.5-0.6 \mathrm{mg} / \mathrm{kg}$ will allow for good intubating conditions within 60 seconds and complete recovery within 5-8 minutes. Smaller doses of succinylcholine may successfully treat an early presentation of laryngospasm before there is significant oxygen desaturation. However, if a second dose of succinylcholine is needed, life-threatening bradycardia may result and pretreatment with atropine is mandatory. Succinylcholine has been the standard emergency neuromuscular blocking agent in the past, but its presence in the office necessitates having the drugs, supplies, and expertise needed to initially manage an episode of malignant hyperthermia $(\mathrm{MH})$. The recognition and management of malignant $\mathrm{MH}$ are not simplistic, and the cost for all the drugs and supplies is quite high.

Rocuronium has a wide dosing range, but at a dose of $1.2 \mathrm{mg} / \mathrm{kg}$, it allows for good intubating condition in approximately 60 seconds. This provides a similar time to good intubating conditions comparable to succinylcholine. Paralysis would, however, last considerably longer than succinylcholine-approximately 45 to 60 minutes, although reversal with neostigmine and glycopyrrolate could likely occur in less than 30 minutes. But with the availability of sugammadex when high doses (16 $\mathrm{mg} / \mathrm{kg}$ ) are used, full reversal occurs within approximately 3 minutes. If intubation is unsuccessful and a "cannot intubate/cannot ventilate" scenario occurs, this may be a shorter duration of action than succinylcholine. Cost is improved over MH drugs at this dose (about \$350 for a 70-kg person currently), and the risk of $\mathrm{MH}$ is not present. It should be noted that sugammadex also interferes with most hormonal contraceptives, and women of childbearing age should be appropriately warned of this interaction.

It is not clear to this author that rocuronium is now the drug of choice for dental office emergency intubation and treatment of laryngospasm. Fortunately, the incidence of $\mathrm{MH}$ with succinylcholine alone, without the presence of potent inhalation anesthetics, is quite low. Succinylcholine is well known in oral and maxillofacial surgeon and dentist anesthesiologist practices, and it has a long history of successful use. Of course, having any emergency paralyzing drugs available is only as good as understanding how and when to use them, having all of the other emergency equipment immediately available, and keeping current in airway management skills. For the time being, succinylcholine is still an appropriate medication for office-based airway emergencies, but rocuronium/sugammadex may also be appropriate. As medicine evolves, it may be that succinylcholine will gradually be displaced by rocuronium and sugammadex or yet another newer, as yet undeveloped agent may render all of these medications obsolete for emergency airway management.

I look forward to the evolution of anesthesia practice and the opportunity to use new drugs and techniques that will improve our patients' surgical experience.

Steven Ganzberg, DMD, MS Editor-in-Chief, Anesthesia Progress 